\title{
Hadronic Final States at HERA
}

Artem Baghdasaryan

Deutsches Elektronen-Synchrotron, Yerevan Physics Institute

\begin{abstract}
The hadronic final state in deep-inelastic ep scattering (DIS) is investigated at HERA. Charged particle production is measured as a function of the pseudorapidity and the transverse momentum, thus testing models of hadroproduction. Scaled momentum distributions of $K_{S}^{0}$ and $\Lambda$ are measured as a functions of the hardscale $Q^{2}$ and are used to test predictions based on fragmentation functions. The production of very forward photons and neutrons is also measured, probing hadroproduction from the proton remnant.
\end{abstract}

Keywords:

2000 MSC: Charged Particle Spectra, Parton-shower, Forward Neutron Production, Forward Photon Production

\section{Charged particle spectra}

To investigate the cascade dynamics, charged particle densities as a function of transverse momentum and pseudorapidity were measured in semi-inclusive DIS $e p \rightarrow e^{\prime} h X$ using the $\mathrm{H} 1$ detector at DESY at centreof-mass energies $\sqrt{s}_{e p}=319 \mathrm{GeV}$ [1] in the kinematic range of low photon virtuality $Q^{2}\left(5<Q^{2}<100 \mathrm{GeV}^{2}\right)$ and small Bjorken $x\left(10^{-4}<x<10^{-2}\right)$. In order to distinguish hadronisation effects from effects due to parton evolution the measurements are divided into two regions: low $p_{T}^{*}\left(0<p_{T}^{*}<1 \mathrm{GeV}\right.$, predominantly sensitive to hadronisation effects) and high $p_{T}^{*}$ $\left(1<p_{T}^{*}<10 \mathrm{GeV}\right.$, predominantly sensitive to parton dynamics). The $p_{T}^{*}$ dependence of the charged particle densities is studied in two different pseudorapidity intervals $0<\eta^{*}<1.5$ and $1.5<\eta^{*}<5$, referred to as the "central region" and "current region" respectively. The target region, $\eta^{*}<0$, is not accessible in this analysis. In figure 1 the charged particle densities are shown as a function of $\eta^{*}$ for $p_{T}^{*}<1 \mathrm{GeV}$ and $1<p_{T}^{*}<10 \mathrm{GeV}$ with the predictions of the DGLAP-like model RAPGAP, using different PDF sets. In the soft $p_{T}^{*}$ region all NLO PDFs (HERAPDF1.0 [2], CTEQ6.6 [3], GRV98NLO [4]) show similar results and predict less particles compared to calculations using the
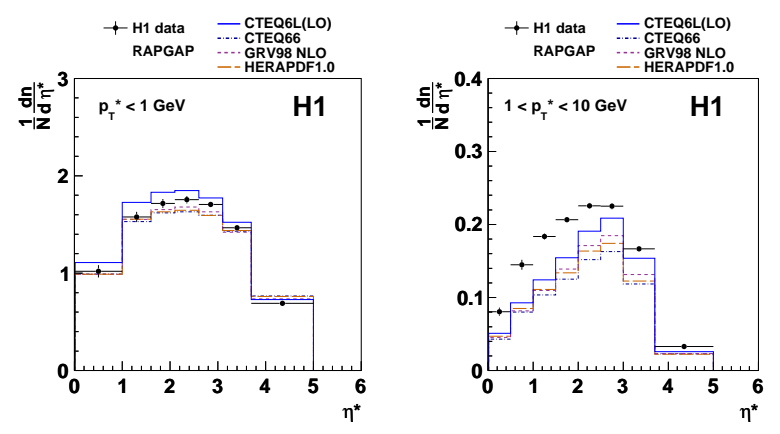

Figure 1: Charged particle density as a function of $\eta^{*}$ for $0<p_{T}^{*}<$ $1 \mathrm{GeV}$ (left) and for $1<p_{T}^{*}<10 \mathrm{GeV}$ (right), compared to RAPGAP predictions with different proton PDFs.

default LO PDF set CTEQ6L(LO). All predictions are close to the data. At large $p_{T}^{*}$ some differences between the NLO PDF sets are observed, with CTEQ6L(LO) being closest to the data, although the differences to the data are still larger than the differences between the various PDF predictions. Similar PDF uncertainties are observed when using the CDM model as implemented in DJANGOH. To check the sensitivity to hadronisation effects, the RAPGAP predictions obtained with three sets of fragmentation parameters (ALEPH tune [5], Pro- 

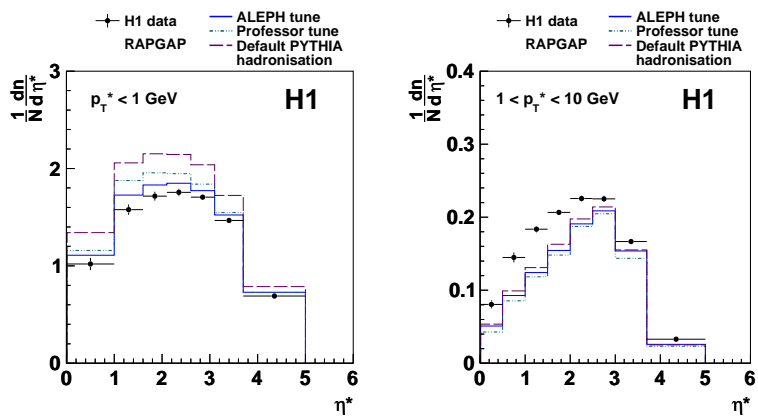

Figure 2: Charged particle density as a function of $\eta^{*}$ for $0<p_{T}^{*}<$ $1 \mathrm{GeV}$ (left) and for $1<p_{T}^{*}<10 \mathrm{GeV}$ (right), compared to RAPGAP predictions for three different sets of fragmentation parameters.

fessor tune [6] and default PYTHIA6.424) are compared to the data in figure 2. Significant differences between these three settings are seen in the soft $p_{T}^{*}$ region, where the data are best described by the ALEPH tune. At large transverse momenta they give similar results but none of them describes the data.

In figure 3 the data are compared to different approaches for QCD radiation: the CDM model DJANGOH [7], the DGLAP-based MC RAPGAP [8] and Herwig++ [9] and the CCFM model CASCADE [10]. In the soft $p_{T}^{*}$ region DJANGOH and RAPGAP describe the data within the PDF uncertainties. Herwig++, which uses the cluster fragmentation model, provides a reasonable description of the data. CASCADE predicts too high multiplicities in most of the measured range. In the region of $1<p_{T}^{*}<10 \mathrm{GeV}$ large difference between the predictions are obtained, and the best description of the data is achieved by DJANGOH. RAPGAP strongly undershoots the data in the central region. Herwig++ predicts a spectrum which is even below the prediction of RAPGAP. CASCADE is significantly above the data in a wide range of $\eta^{*}$.

In figure 4 the charged particle densities as a function of $p_{T}^{*}$ are shown for two pseudorapidity intervals $0<\eta^{*}<$ 1.5 (central) and $1.5<\eta^{*}<5$ (current). The shapes of the measured $p_{T}^{*}$ distributions in the two pseudorapidity ranges are similar. Predictions based on different parton shower dynamics differ significantly at high $p_{T}^{*}$ : RAPGAP and HERWIG++ (DGLAP) strongly undershoots the data not only in the central but also in the current region, DJANGOH (CDM) provides the best description of experimental data in both $p_{T}^{*}$ and $\eta^{*}$ regions but still not good.

Charged particle production is investigated at $\mathrm{H} 1$ also at $\sqrt{s}_{e p}=225 \mathrm{GeV}$ [11]. At this centre-of-mass energy, using the phase space $5<Q^{2}<10 \mathrm{GeV}^{2}$ and
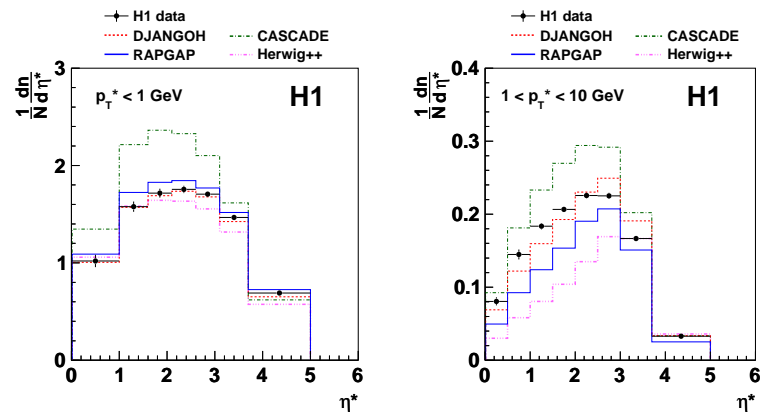

Figure 3: Charged particle density as a function of $\eta^{*}$ for $0<p_{T}^{*}<$ $1 \mathrm{GeV}$ (left) and for $1<p_{T}^{*}<10 \mathrm{GeV}$ (right), compared to MCs predictions.
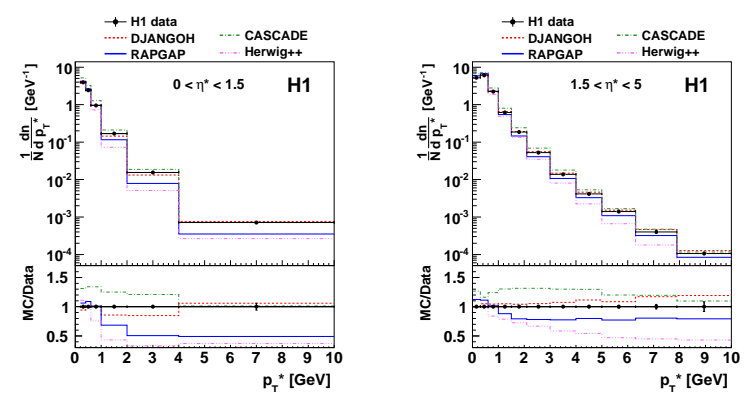

Figure 4: Charged particle density as a function of $p_{T}^{*}$ in $0<\eta^{*}<1.5$ (left) and $1.5<\eta^{*}<5$ (right) regions, compared to MCs predictions.

$0.35<y<0.8$, an improved acceptance and momentum resolutions is achieved for $0<\eta^{*}<3.5$. Cross sections are measured double-differential in $\eta^{*}$ and $p_{T}^{*}$. The shape of the cross section as a function of pseudorapidity is modeled well by the DJANGOH and RAPGAP Monte Carlo generators. However, neither Djangoh nor Rapgap describes the charged particle densities as a function of transverse momentum $p_{T}^{*}$, when measured in $\eta^{*}$ bins. This confirms the results discussed above.

To study changes in the hadroproduction dynamics along $\eta^{*}$, a phenomenological model is used. This model [12] describes the $p_{T}^{*}$ spectrum as the sum of an exponential (Boltzmann-like) and a power-law statistical distribution. It provides a much better description of the data than the often used Hagedorn parameterisation [13]. The relative contribution of the exponential and power-law terms of the model can be characterized by ratio $R$ of the power-law term alone to the total contribution. In figure 5 the relative contribution $\mathrm{R}$ of the power-law type distribution to the charged particle production spectra is shown as function of the charged particle rapidity $\eta^{*}$. Close to the virtual photon direction 
(large values of $\eta^{*}$ ) the $p_{T}^{*}$ spectrum can be described

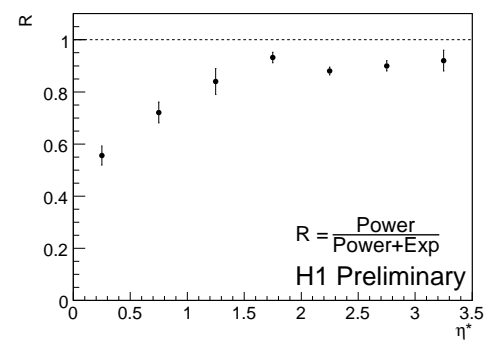

Figure 5: The relative contribution of power-law term.

by a power-law term only, while at central rapidities the data require a significant exponential contribution.

\section{Scaled momentum distributions for in DIS}

The data most widely used to extract fragmentation functions (FFs) comes from $e^{+} e^{-}$annihilations into charged hadrons. These data are very precise and the predicted cross sections do not depend on PDFs. However, they do not provide information on how to disentangle quark and anti-quark contributions to the FFs and also the gluon fragmentation remains largely unconstrained. For further constraining FFs, scaled momentum distributions, $(1 / N)\left(n(H) / \Delta x_{p}\right)$, with $n(H)$ the number of $H$ ( $K$ or $\Lambda), \mathrm{N}$ the number of DIS events in a given $Q^{2}$ bin and $\Delta x_{p}$ the width of the $x_{p}$ bin, were measured in the current region of the Breit frame [14]. The distributions are presented as functions of $Q^{2}$ and scaled momentum $x_{p}\left(x_{P}=2 * P_{\text {Breit }} / \sqrt{Q}^{2}\right)$ in the kinematic region of $10<Q^{2}<40000 \mathrm{GeV}^{2}$ and $0.001<x<0.75$. Figure 6 shows the scaled momentum distributions for $K_{S}^{0}$ as functions of $Q^{2}$ in different regions of $x_{p}$. The data show clear scaling violations. This behavior is qualitatively predicted by the parton evolution with increasing $Q$ : the phase space for soft gluon radiation increases, leading to a rise of the number of soft particles with small $x_{p}$. The measurements have the potential to constrain significantly the FFs for the strange hadrons $K_{S}^{0}$ and $\Lambda / \bar{\Lambda}$.

\section{Forward Neutron and Photon Production in DIS}

Forward photon and neutron production cross sections $\left(\eta^{L a b}>7.9\right)$ are measured as a function of the energy $W$ of the virtual photon-proton system in HCM in the kinematic range $6<Q^{2}<100 \mathrm{GeV}^{2}$ and $0.05<$ $y<0.6$ [17]. In Figure 7 normalised to DIS photon and neutron production cross sections as a functions of $x_{F}$
ZEUS
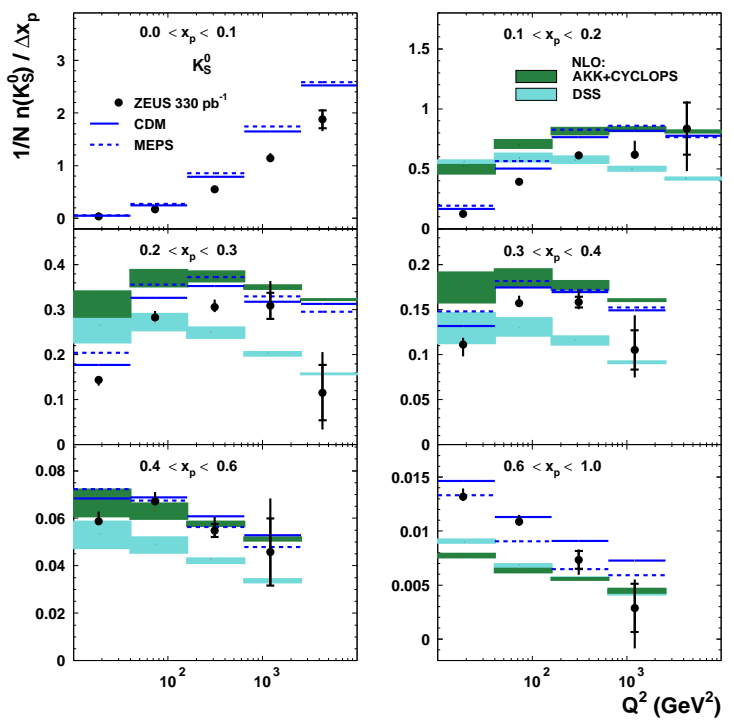

Figure 6: The measured scaled momentum distributions as functions of $Q^{2}$ in different regions of $x_{p}$ (dots). The inner error bars represent the statistical uncertainty. The outer error bars show the statistical and systematic uncertainties added in quadrature. The predictions from CDM [15] (solid lines) and MEPS [16] (dashed lines) are also shown.

in different $W$ intervals are compared to the MC predictions. Photon data are significantly lower than predicted by $\mathrm{MC}(\mathrm{Data} / \mathrm{CDM}=0.6)$ and neutron data are larger than fragmentation contribution (Data/CDM $=1.35)$. In Figure 8 the same data are compared to the models of the Cosmic Ray hadronic interactions (CR): EPOS [18], SIBYLL [19], [20] and two versions of QGSJET [21], [22]. Large difference between CR models predictions observed and none of the models describes the photon and neutron data simultaneously. In order to test the Feynman scaling, photon production cross sections at different energy values $(70<W 1<130 \mathrm{GeV}$, $130<W 2<190 \mathrm{GeV}, 190<W 3<250 \mathrm{GeV})$ are compared. In the figure 9 are shown the cross sections' ratio as well as MCs (upper) and CR models (lower) predictions. The data are consistent with being constant within error, this supporting the hypothesis of Feynman scaling. In contradiction, CR models show W dependence.

\section{Conclusion}

The charged hadron spectra at low $Q^{2}$ and small $x$ are measured in DIS. The data are compared to QCD models with different evolution approaches for simu- 


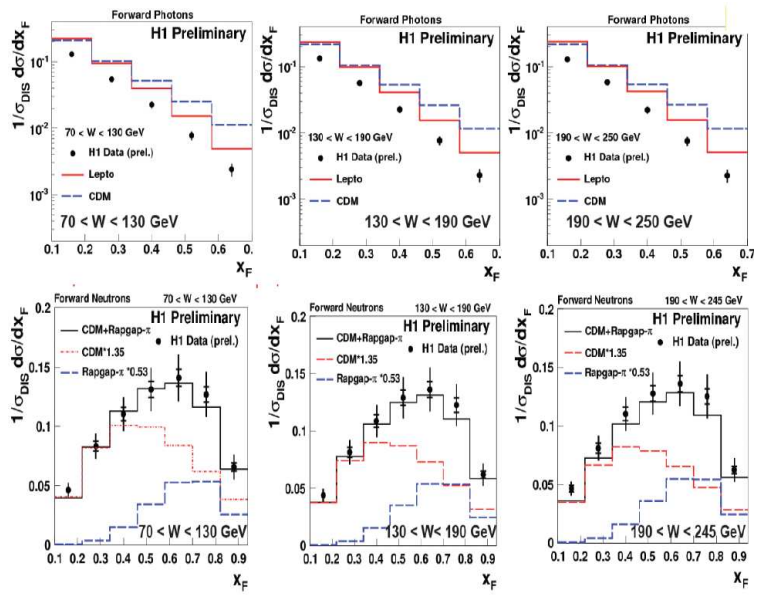

Figure 7: Normalised to DIS photon and neutron production cross sections, compared to MC-s.
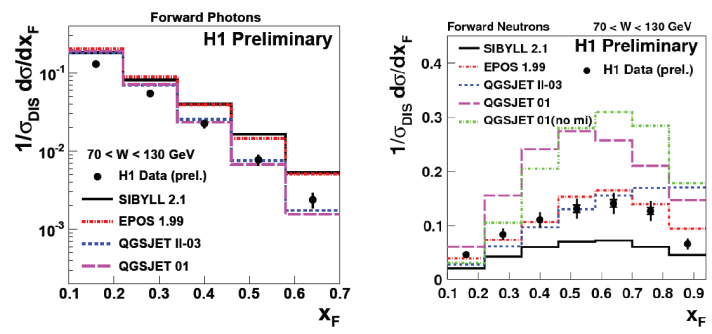

Figure 8: Normalised to DIS photon and neutron production cross sections, compared to hadronic interaction models.

lating the parton cascade and with different hadronisation schemes The Colour Dipole Model implemented in DJANGOH is the best among the other considered MC models and provides a reasonable description of the data, but not good. Phenomenologycal model with a significant exponential contribution in the central region provide a good description of the data.

Scaled momentum distributions for $K_{S}^{0}$ and $\Lambda$ hadrons are measured for the first time in $e p$ DIS. NLO QCD calculations, based on different parametrisations of FFs fail to describe the measurements. The measurements have the potential to constrain significantly the FFs for the strange hadrons $K_{S}^{0}$ and $\Lambda$.

Forward photon and neutron production cross sections are measured. The measurements are compared to the $\mathrm{MC}$ and Cosmic Ray interaction models. None of the models describes photon and neutron data simultaneously well.

\section{References}

[1] C. Alexa et al., H1 Collab., Eur. Phys. J., C73, 02/13, 2406
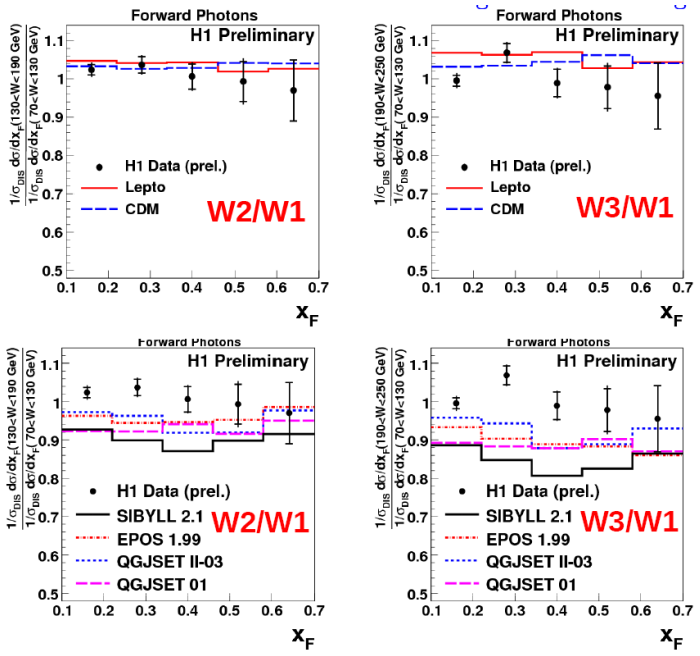

Figure 9: Cross sections ratios at different $W$.

[2] F. Aaron et al., H1 and ZEUS Collab., JHEP 1001, (2010), 109

[3] P.M. Nadolsky et al., Phys. Rev. D78 (2008) 013004

[4] M. Gluck, E. Reya and A. Vogt, Eur. Phys. J. C5 (1998) 461

[5] S. Schael et al., Phys. Lett. B606 (2005) 265

[6] A. Buckley et al., Eur. Phys. J. C65 (2010) 331

[7] K.Charchula et al., DJANGOH 1.4, Comput.Phys.Commun.81 (1994) 381

[8] H. Jung, RAPGAP 3.1, Comput. Phys. Commun 86 (1995) 147

[9] S. Gieseke et al., Herwig++ 2.5, [arXiv:1102.1672]

[10] H. Jung, Comput. Phys. Commun. 143 (2002) 100

[11] H1 prelim-13-032

[12] A. A. Bylinkin and A. A. Rostovtsev, Phys. Atom. Nucl. 75 (2012) 999

[13] R. Hagedorn, Riv. Nuovo Cim. 6 (1983) 1

[14] H. Abramowicz et al., ZEUS Collab., JHEP 03 (2012) 020

[15] B. Andersson et al., Z. Phys. C 43 (1989) 625

[16] G. Ingelman et al., Comput. Phys. Commun. 101 (1997) 108

[17] H1 prelim-13-012

[18] K.Werner et.al., Phys.Rev. C74 (2006) 044902

[19] J.Engel et al., Phys.Rev. D46 (1992) 5013

[20] E.-J.Ahn et al., Phys.Rev. D80(2009)094003

[21] N.N.Kalmykov et.al., Nucl.Phys.Proc.Suppl.52B (1997) 17

[22] S.S.Ostapchenko, AIP Conf.Proc.928 (2007) 118 